\title{
Epidural anaesthesia and long term backache after childbirth
}

\author{
C MacArthur, M Lewis, E G Knox, J S Crawford
}

\begin{abstract}
Objective-To examine the relation between epidural anaesthesia and long term backache after childbirth.

Design-Data from postal questionnaire on morbidity after childbirth sent to women who had delivered in one maternity hospital between 1978 and 1985 were linked to maternity case notes for each woman.

Setting - Maternity hospital in Birmingham.

Subjects-11 701 Women who had delivered their most recent baby at the maternity hospital during the defined period and who returned their completed questionnaires.

Main outcome measures and results - Of the 1634 women who reported backache, $1132(69 \%)$ had had it for over a year. A significant association was found between backache and epidural anaesthesia (relative risk=1.8); 903 of 4766 women (18.9\%) who had had epidural anaesthesia reported this symptom, compared with 731 of the 6935 women $(10.5 \%)$ who had not had epidural anaesthesia. This association was consistent in both "normal" and "abnormal" deliveries, the only exception being after an elective caesarean section when no excess backache occurred after epidural anaesthesia.

Conclusions - The relation between backache and epidural anaesthesia is probably causal. It seems to result from a combination of effective analgesia and stressed posture during labour. Further investigations on the mechanisms causing backache after epidural anaesthesia are required.
\end{abstract}

Department of Social

Medicine, Medical School, University of Birmingham, Birmingham B15 2TJ

C MacArthur, PHD, research fellow

E G Knox, MD, professor

Birmingham Maternity Hospital, Queen Elizabeth Medical Centre, Birmingham B15

$M$ Lewis, FCANAES, consultant anaesthetist J S Crawford, FCANAES, formerly consultant anaesthetist

Dr Crawford died in August 1988.

Correspondence to:

Dr MacArthur.

BrMed f 1990;301:9-12

\section{Introduction}

Epidural anaesthesia has been widely used over the past 20 years for pain relief in labour and more recently as an alternative and preferred form of anaesthesia for caesarean sections. In the maternity hospital where this study was carried out 33500 epidurals had been given up to the time of our inquiry, all patients being monitored daily by the anaesthetists until they were discharged from hospital. Much information is therefore available from this series and from others concerning immediate side effects. A very small proportion of women have serious side effects (total spinal block, severe hypotension, accidental intravenous injections) and others experience less serious effects (mild hypotension, shivering) during epidural anaesthesia. ${ }^{1-3}$ Headache, backache, or bladder problems occur occasionally in the following few days..$^{3-5}$ These effects have all been considered transient, having diminished or disappeared by the time of hospital discharge.

No investigations have, however, been published concerning the possible existence of longer term problems. This is probably because no real concern had ever arisen, either on theoretical grounds or from anecdotal commentaries by patients or obstetricians.
Nevertheless, for such a frequently and routinely used procedure we considered it desirable to establish whether or not epidural anaesthesia is indeed free from long term complications.

\section{Subjects and methods}

This report is part of a wider investigation of long term morbidity after childbirth (to be published by HMSO). This is a subject that has rarely been examined in a systematic way. The broad objectives of our larger investigation were, firstly, to ascertain the nature and prevalence of morbidity after childbirth and, secondly, to examine its relation with a wide range of obstetric, anaesthetic, and maternal factors. One focus of interest was epidural anaesthesia.

The study population consisted of 11701 women who had delivered their most recent child in Birmingham Maternity Hospital between 1978 and 1985. As the inquiry was undertaken in January 1987 all deliveries had occurred more than 12 months before this date, the longest follow up period being nine years.

There were two sources of data. Information about morbidity was obtained from a postal questionnaire sent to the women. Twenty five different health problems were listed. For each, the women were asked whether they had experienced the problem since delivering the index child (date of birth specified on the form); if so, how soon after the birth had the symptom occurred; when had it stopped; had they had it before; and had they sought medical advice. Care was taken in the questionnaire and covering letter to provide no indication that any specific obstetric or anaesthetic procedures were being investigated. The second source of data was the computerised maternity casenotes assembled for every woman, which provided information on all the obstetric and anaesthetic factors as well as the basic social characteristics of the mother. This file also supplied names and addresses for the postal inquiry. The data sources for each woman were subsequently linked.

The addresses from the case notes were often in correct as many women had moved in the intervening years. It would have been possible to update addresses using family practitioner committee records, but we were refused access to them so an alternative approach had to be devised. A pilot group of 250 women was sent questionnaires using the addresses obtained from casenotes, and $88(35 \%)$ returned them completed. Addresses of the remaining 162 were checked in the electoral registers and with the Post Office; only 22 of these women still resided at their original address. In all, 110 could thus be presumed to have received their questionnaires, giving an estimated response rate of $80 \%$. In the main study almost $40 \%$ (11701) of the 30096 women who had delivered at the hospital during the defined period returned their questionnaires completed. A further check in the manner described above of 1000 addresses of women who did 
not return questionnaires confirmed an acceptable response rate of $79 \%$ from those still living at the same address. Response bias other than migration bias was therefore likely to be minimal.

We had full obstetric case notes for all 30096 women. Comparisons between those returning and not returning questionnaires showed that the groups had similar obstetric and anaesthetic characteristics, with $41 \%$ and $37 \%$ respectively having had epidural anaesthesia. The groups had some differences in their maternal characteristics, however, with more of the women not returning questionnaires being younger, single, Asian, and of lower social class. These groups were subsequently discovered to report higher rates of backache; thus a greater proportion of such groups in the sample would have led to an even higher frequency of backache.

We describe a relation discovered between epidural anaesthesia and backache among the 11701 subjects. For this purpose, 4766 women who had this procedure were compared with 6935 who did not.

\section{Results}

Long term backache after childbirth was commonly reported in this population. Almost a quarter of the women $(23.3 \% ; 2730)$ reported backache occurring within three months of their delivery and persisting for more than six weeks. Many of these women (1096) had also had backache on a previous occasion. As the main objective of this study was to examine the relation between symptoms (in this case backache) and the obstetric and anaesthetic characteristics of a nominated delivery, our main analysis related only to newly occurring backache. There were 1634 women in the population $(14.0 \%)$ who reported having backache that they had never had before, which had begun within three months of their delivery and which had lasted more than six weeks. All the analyses of backache described here relate to these 1634 women. (We distinguished in the questionnaire between middle and lower backache and the analysis was conducted for each separately. The results were substantially the same so the two are combined.)

Although all symptoms that lasted more than six weeks were considered to be of sufficient duration to be included, most backaches had lasted considerably longer than this. Of the 1634 women who reported backache, $69 \cdot 3 \%(1132)$ had had it for over a year, and $65.0 \%(1062)$ said that they still had it at the time of questioning. Many of these women were delivered several years previously. Persistent backache was reported by women who had delivered in the earlier and in the later years in equal proportions. A substantial number of these backaches must therefore have developed into chronic conditions.

Several of the obstetric, anaesthetic, and maternal factors examined in the study were found to be statistically associated with subsequent backache. There was a highly significant association with epidural anaesthesia: $18.9 \%(903 ; 95 \%$ confidence interval $15 \cdot 5 \%$ to $21 \cdot 5 \%$ ) of the women who had this procedure reported newly occurring backache beginning within three months of the delivery and persisting for more than six weeks, compared with $10 \cdot 5 \%(731 ; 8 \cdot 3 \%$ to $12.7 \%$ ) of those who had not had epidural anaesthesia. Many of the factors, however, were statistically associated with each other, and the interpretation of simple associations between pairs of factors was problematical. A form of multivariate analysis was necessary to take such complex interrelations into account. The most appropriate form of multivariate analysis in this instance was discriminant analysis; it was carried out with the statistical package for social sciences (SPSS X). This procedure takes simultaneous account of a large number of variables, and calculates which of them independently and significantly predict a woman having a particular symptom. The discriminant analysis procedure examined and selected variables in a stepwise manner, the most significant association being selected first, then the next significant, and so on. Factors were entered in continuous format wherever relevant (for example, age and duration of labour) and otherwise in binary categorical format.

The discriminant analysis showed that, of the factors associated with backache, epidural anaesthesia was by far the most strongly predictive. Women who had epidural anaesthesia were much more likely to suffer from subsequent newly occurring long term backache even when allowance was made for all the other associated factors (table I).

Epidural anaesthesia is more often given when there are difficult circumstances before delivery (such as atypical fetal presentations) and is associated with less straightforward deliveries (longer second stage, forceps delivery, etc). The standardisation effected through discriminant analysis controls the effects of such associations, and it showed that these other high risk characteristics were not themselves directly responsible for the subsequent backache; the prediction seemed to be almost entirely a function of having epidural anaesthesia.

We nevertheless retested the relation in more explicit detail and in several different ways. First we identified a group of women who had "normal" straightforward deliveries and compared the frequency of backache with and without epidural anaesthesia in this group alone. A normal delivery was defined here as a singleton with an occipitoanterior presentation at delivery, a spontaneous onset of labour, no forceps, no caesarean section, an active phase of labour of less than 12 hours, and a second stage of under two hours. We then repeated the epidural comparisons for the "abnormal" deliveries (table II). This showed that

TABLE II - "Normal" deliveries, epidural anaesthesia, and backache

\begin{tabular}{|c|c|c|}
\hline & $\begin{array}{l}\text { No (\%) with } \\
\text { backache }\end{array}$ & $\begin{array}{l}95 \% \\
\text { Confidence } \\
\text { interval }\end{array}$ \\
\hline $\begin{array}{l}\text { "Normal" delivery: } \\
\text { With epidural }(n=1098) \\
\text { Without epidural }(n=4646)\end{array}$ & $\begin{array}{l}200(18 \cdot 2) \\
474(10 \cdot 2)\end{array}$ & 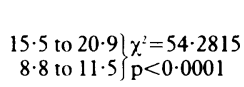 \\
\hline $\begin{array}{l}\text { "Abnormal" delivery: } \\
\text { With epidural }(n=3668) \\
\text { Without epidural }(n=2289)\end{array}$ & $\begin{array}{l}703(19 \cdot 2) \\
257(11 \cdot 2)\end{array}$ & $\begin{array}{c}17 \cdot 2 \text { to } 20 \cdot 7 \\
9 \cdot 2 \text { to } 13 \cdot 2\} \chi_{\mathrm{p}}^{2}=65 \cdot 0001122\end{array}$ \\
\hline
\end{tabular}

irrespective of whether the delivery was normal or abnormal those women who had had epidural anaesthesia reported a significant excess of subsequent backache. In fact, the normality of the delivery was not itself related to subsequent backache. The crude relative risk of backache after epidural anaesthesia, taking no account of the normality of delivery, was 1.80. Among women with normal deliveries, the relative risk associated with epidural anaesthesia was 1.79; among women with abnormal deliveries it was $1 \cdot 71$.

We next examined the possibility that the epiduralbackache association might be an artefact arising from varying pain thresholds. Some women may choose to have epidural anaesthesia because they have a low pain tolerance: either they are less able to withstand pain or they complain more of it. These women would then be more likely to complain of subsequent back pain. The association between epidural anaesthesia and back pain might thus reflect nothing more than a correlated propensity to complain of pain on separate occasions.

There was no explicit information in the case notes of why the women had epidural anaesthesia: whether
TABLE I - Discriminant ana
of factors associated with rackache. $F$ value indicates factor to discriminant function

\begin{tabular}{lc}
\hline Factors & F value \\
\hline Epidural anaesthesia & 83.789 \\
Nulliparity & 29.688 \\
Asian race & 23.392 \\
Younger maternal age & 16.07 \\
No episiotomy & 9.969 \\
Spinal anaesthesia & 7.731 \\
Longer second stage labour & 6.439 \\
Spontaneous onset of & \\
$\quad$ labour & 5.267 \\
Social class III, IV, V & 4.032 \\
\end{tabular}


they chose it themselves simply for pain relief or whether they were advised to have it for medical reasons. However, we identified several circumstances before delivery that were thought likely to precipitate such advice, and we showed that these "indications" were indeed associated with a higher rate of epidural anaesthesia. These medical indications comprised maternal hypertension, an induced labour, atypical fetal presentation, or a multiple pregnancy. If the hypothesis of low pain threshold were valid we would expect a reported excess of subsequent backache only in women without these indications-that is, in the group that chose epidural anaesthesia just for pain relief. In fact there were no differences between these groups, providing no evidence that the association might result from the existence of a group of "persistent complainers."

Our findings relating to caesarean section provided important insights into the possible mechanism of postepidural backache. In this population $16.4 \%$ (1915) of the women delivered their babies by caesarean section; $59.8 \%$ of these (1145) were emergency procedures, the remainder being elective procedures with no preceding labour. Comparing the effects of epidural anaesthesia on subsequent rates of backache in each of these types of delivery we found that the same epidural-backache relations occurred after an emergency caesarean section as after a vaginal delivery. After an elective caesarean section, however, the backache rate did not differ whether the woman had epidural or general anaesthesia (table III). It would seem then that excess backache was determined by a particular combination of events: epidural anaesthesia combined with a period of time in labour.

TABLE III - Type of delivery, epidural anaesthesia, and backache

\begin{tabular}{|c|c|c|}
\hline & $\begin{array}{l}\text { No (\%) with } \\
\text { backache }\end{array}$ & $\begin{array}{l}95 \% \\
\text { Confidence } \\
\text { interval }\end{array}$ \\
\hline $\begin{array}{l}\text { Vaginal delivery: } \\
\text { With epidural }(n=3688) \\
\text { Without epidural }(n=6098)\end{array}$ & $\begin{array}{l}713(19 \cdot 3) \\
637(10 \cdot 4)\end{array}$ & $\begin{array}{c}17 \cdot 8 \text { to } 20 \cdot 7 \mid \chi^{2}=151 \cdot 8790 \\
9 \cdot 2 \text { to } 11 \cdot 6\} \\
\mathrm{p}<0.001\end{array}$ \\
\hline $\begin{array}{l}\text { Emergency caesarean section: } \\
\text { With epidural }(n=652) \\
\text { Without epidural }(n=493)\end{array}$ & $\begin{array}{r}131(20 \cdot 1) \\
56(11 \cdot 4)\end{array}$ & $\begin{array}{r}16.5 \text { to } 23.5 \\
7.1 \text { to } 15.5\} \\
\chi^{2}=15.0357\end{array}$ \\
\hline $\begin{array}{l}\text { Elective caesarean section: } \\
\text { With epidural }(n=426) \\
\text { Without epidural }(n=344)\end{array}$ & $\begin{array}{l}59(13 \cdot 8) \\
38(11 \cdot 0)\end{array}$ & $\begin{array}{l}9 \cdot 3 \text { to } 18 \cdot 3 \\
5.6 \text { to } 15 \cdot 4 \\
\chi^{2}=1 \cdot 11157\end{array}$ \\
\hline
\end{tabular}

The other independent non-epidural associations with backache shown in table I also require comment. Firstly, the effect of primiparity is probably an artefact arising from the selective exclusion of certain women with higher parity. This is because we have included among those affected only the women with no previous backache; multiparous women have had greater previous opportunity of having backache, so their current backache would be excluded. The effect for maternal age probably originates for analogous reasons. When discriminant analysis was completed using only first births the effect of age disappeared, confirming that it was probably an artefact. The higher risk of backache in Asian mothers, however, does not arise from such causes and deserves additional investigation.

The other associations shown in table I were much weaker. The relation with spinal anaesthesia is consistent with the epidural effect and we must consider common mechanisms. The association between backache and prolonged second stage labour could result from stressed postures during the second stage, which might damage the back if they are unrelieved for long periods. These associations between backache and other factors will be examined in more detail in additional publications and some are being pursued in further investigations.

\section{Discussion}

This investigation shows a clear association between epidural anaesthesia and an increased rate of subsequent newly occurring backache, often persisting over many months or years and possibly becoming chronic. The effect was clearly evident in many different circumstances. The hypothesis that the association might be explained indirectly through one or more of the many interrelated confounding factors was tested and not upheld. Enhancement of the risk of backache after epidural anaesthesia was not affected by the normality or otherwise of the delivery, and different reactions to pain also did not provide an explanation. The association with epidural anaesthesia showed the consistency expected from a cause-effect relation.

What might be the mechanism of causation? Is it a consequence of the trauma of needle insertion, or is there some other explanation? The most direct evidence was found in the data relating to caesarean sections. The only group of women with no excess backache after epidural anaesthesia was the group who had an elective caesarean section. These women had not laboured. If the backache had been from direct needle trauma it should have occurred in this group too. Rather it seems that backache results from a combination of anaesthesia together with labour, and it makes no difference whether this is terminated by vaginal delivery or by an emergency caesarean section.

The most plausible hypothesis is that the origin of the problem is postural. Stressed positions can occur in normal labour and do themselves give rise to subsequent backache. Indeed, in this study persistent and newly occurring backache follows in about one in 10 deliveries without epidural anaesthesia. Such postural problems could be exacerbated by the muscular relaxation and abolition of pain associated with epidural anaesthesia. In addition, movement under epidural anaesthesia generally requires assistance, so that a woman could remain without immediate discomfort or complaint for some hours in a potentially damaging position. The additional independent association between backache and a prolonged second stage labour (table I) supports this hypothesis.

In a series of 9107 patients having epidural anaesthesia for surgery not associated with childbirth a subsequent backache rate of only $2 \%$ was recorded. ${ }^{6}$ This very low rate after epidural anaesthesia alone would support our hypothesis that epidural anaesthesia plus labour is necessary to produce the much higher rates of backache found in our study.

This postural hypothesis will guide the next stages of our inquiries. Unfortunately, neither the durations nor the doses of the epidural anaesthetics were recorded in the computerised case notes. These data will, however, be available from a further set of anaesthetic records (for the same women), and we shall investigate the effects of these factors. Additional investigations already underway on a further cohort of women will examine the detailed characteristics of backache (severity, type) after deliveries with and without epidural anaesthesia. After consultation with orthopaedic and physiotherapeutic colleagues, we hope to design a preventive trial of posture management during labour.

How large is the problem that we seem to have uncovered? Irrespective of whether the normal or abnormal deliveries were used in making the calculation, the attributable risk of backache after epidural anaesthesia is 8/100: of every hundred women who have an epidural during labour, eight will develop long term backache as a direct consequence. If, say, $25 \%$ of women delivering in England and Wales are currently receiving epidural anaesthesia then about 13000 women may be affected each year in this way. 
These are not, of course, grounds for condemning epidural anaesthesia. It should not, in Reynold's terms, be "classed with other forms of obstetric intervention as undesirable interference by the medical profession in what would otherwise be a trouble free and satisfying natural event." In addition to providing the only really effective form of pain relief during labour, ${ }^{89}$ an epidural offers a number of positive clinical benefits for both the mother ${ }^{1011}$ and the baby. ${ }^{12-14}$ Moreover, the backache is not solely a consequence of epidural anaesthesia but is probably due to a combination of muscular relaxation and postural stresses in labour. The problem now is to determine in precise detail the mechanisms that result in backache and to refine the management of epidural anaesthesia in labour.

Thanks are due to all women who took part in the study. The study was financed by a grant from the Department of Health.

1 Crawford JS. Some maternal complications of epidural analgesia for labour. Anaesthesia 1985;40:1219-25.
2 Crawford JS. Principles and practice of obstetric anaesthesia. 5th ed. Oxford: Blackwell Scientific, 1985.

3 Ramanathan S. Obstetric anesthesia. Philadelphia: Lea and Febiger, 1988

4 Crawford JS. Lumbar epidural block in labour: a clinical analysis. Br $\mathcal{F}$ Anaesth 1972;44:66.

5 Grove LH. Backache, headache and bladder dysfunction after delivery. Brf Anaesth 1973;45:1147-9.

6 Dawkins CJM. An analysis of the complication of extradural and caudal block. Anaesthesia 1969;24:554.

7 Reynolds F. Epidural analgesia in obstetrics: pros and cons for mother and baby. BrMed f 1989;299:751-2.

8 Morgan BM, Bulpitt CJ, Clifton P, Lewis PJ. The consumers' attitude to obstetric care. Br $\mathcal{F}$ Obstet Gynaecol 1984;91:624-8.

9 Robinson JO, Rosen M, Evans JM, Revill SI, David H, Rees GAD. Matern opinion about analgesia for labour. A controlled trial between epidural block and intramuscular pethidine combined with inhalation. Anaesthesia 1980 35:1173-81.

10 Newsome LR, Bramwell RS, Curling PE. Severe pre-eclampsia: hemodynamic effects of lumbar epidural anesthesia. Anesthesia and Analgesia 1986;65:31-6.

11 Morgan BM, Aulakh JM, Barker JP, Reginald PW, Goroszeniuk T, Trojanowski A. Anaesthetic morbidity following caesarean section under epidural or general anaesthesia. Lancet 1984;i:328-30.

12 David H, Rosen M. Perinatal mortality after epidural anaesthesia. Anaesthesia 1976;31:1054-9.

13 Maltau JM, Egg K. Epidural analgesia and perinatal retinal haemorrhages. Acta Anaesthesiol Scand 1980;24:99-101.

14 Crawford JS. A prospective study of 200 consecutive twin deliveries. Anaesthesia 1987;42:33-43.

(Accepted 23 April 1990)

\title{
Bleeding time in patients with hepatic cirrhosis
}

\author{
J C Blake, D Sprengers, P Grech, P A McCormick, N McIntyre, A K Burroughs
}

Abstract

Objective-To determine the frequency of an abnormal bleeding time in patients with cirrhosis and to relate this to known factors that affect primary haemostasis and to the severity of liver disease.

Design-Prospective clinical and laboratory study in patients admitted for complications or investigations of liver disease.

Setting-Royal Free Hospital hepatobiliary and liver transplantation unit.

Subjects - 100 Consecutive inpatients aged 17-74 with various forms of cirrhosis, including alcoholic, biliary, autoimmune, viral, and cryptogenic. At least 10 days had elapsed since any episodes of bleeding, resolution of sepsis, or alcohol intake. No patient was taking any drug known to affect primary haemostasis.

Main outcome measures-Bleeding time as measured with the Simplate double blade template device. A bleeding time longer than $\mathbf{1 0}$ minutes was considered abnormal. Other measures were platelet count, prothrombin time, partial thromboplastin time, packed cell volume, and blood urea, serum bilirubin, and serum albumin concentrations, all measured on each subject at the same time by standard laboratory methods.

Results - A weak but significant correlation existed between the bleeding time and the platelet count $\left(r_{s}=0.483 ; p<0.001\right)$. There were significantly lower platelet counts, longer prothrombin times, and higher blood urea and serum bilirubin concentrations in the $\mathbf{4 2}$ patients with bleeding times of 10 minutes or more compared with the 58 patients with bleeding times less than 10 minutes. Multiple linear regression analysis showed that the bilirubin concentration as well as the platelet count was independently correlated with the bleeding time. The combination of a platelet count greater than $80 \times 10^{9} / 1$ and a prothrombin time less than 17 seconds (usually taken as safe limits for performing routine liver biopsy) did not predict a normal bleeding time. Ten of 39 patients fulfilling these criteria had a prolonged bleeding time.
Conclusions - Prolonged bleeding time is common in patients with cirrhosis, even in those with prothrombin times and platelet counts within "safe limits" for invasive procedures. The severity of liver disease as assessed by the bilirubin concentration plays an important part in determining the bleeding time in cirrhosis. The bleeding time should be measured when assessing patients for invasive procedures who have a raised bilirubin concentration or poor hepatic function, even if the platelet count and prothrombin time are considered adequate.

\section{Introduction}

The bleeding time is an easily performed, frequently used test of primary haemostasis and a reliable indicator of platelet function..$^{1-3} \mathrm{~A}$ survey in the United Kingdom found that $88 \cdot 5 \%$ of hospital haematology departments used the bleeding time, and half used the "template bleeding time" modification of the Ivy test to assess platelet function. ${ }^{3}$ The upper limit of the normal range varies with the operator and the technique employed Most users of the Simplate template device (General Diagnostics, Organon Teknika) take the upper norma limit as between 7 and 10.5 minutes. The commonest normal limit is between 9 and 10 minutes. ${ }^{3}$ The bleeding time is not only influenced by the platele count but may also be affected by the packed cel volume, blood urea concentration, platelet volume, and nature of the connective tissue. Uraemia is associated with an increased bleeding tendency and is also associated with a prolonged bleeding time. ${ }^{+}$Harker and Slichter showed that in thrombocytopenia due to decreased platelet production the bleeding time is inversely correlated with platelet counts below $100 \times$ $10^{9} / 1 \quad(r=-0.971 ; p<0.001) .^{5}$ They also found this relation in 11 patients with hypersplenism, but they included only four patients with cirrhosis in their study.

Although severe thrombocytopenia is reportedly unusual in patients with cirrhosis, ${ }^{6-8}$ platelet counts below $100 \times 10^{9} / 1$ are common. Standard texts of hepatology and haematology do not discuss the bleeding 